\title{
LOW LEVEL LASER THERAPY (LLLT) FOR PATIENTS WITH SACROILIAC JOINT PAIN
}

\author{
Ikuko Ohkuni ${ }^{1}$, Nobuyuki Ushigome ${ }^{1}$, Takashi Harada ${ }^{1}$, Toshio Ohshiro ${ }^{2}$ \\ Kazuhiro Mizutani ${ }^{3}$,Yoshiro Musya ${ }^{3}$,Yukihiko Okada ${ }^{4}$, Hiroshi Takahashi ${ }^{5}$ \\ 1: Dept. of Rehabilitation Medicine, Toho University School of Medicine \\ 2: Japan Medical Laser Laboratory \\ 3: Dept. of Second Orthopaedic Surgery, Toho University School of Medicine \\ 4: Okada Orthopaedic Clinic, Yokohama, Japan \\ 5: Dept. of Orthopaedic Surgery Toho University School of Medicine
}

\begin{abstract}
Background and Aims: Sacroiliac joint pain not associated with a major etiological factor is a common problem seen in the orthopedic clinical setting, but diagnosis is difficult because of the anatomical area and thus it is sometimes difficult to effect a complete cure. Low level laser therapy (LLLT) has been well-reported as having efficacy in difficult pain types, so the following preliminary study was designed to assess the efficacy of LLLT for sacroiliac pain.

Materials and Methods: Nine patients participated, 4 males and 5 females, average age of 50.4 yrs, who attended the outpatient department with sacroiliac pain. The usual major disorders were ruled out. Pain was assessed subjectively pre-and post-LLLT on a visual analog scale, and trunk range of motion was examined with the flexion test to obtain the pre- and post-treatment finger to floor distance (FFD). The LLLT system used was an $830 \mathrm{~nm} \mathrm{CW}$ diode laser, $1000 \mathrm{~mW}, 30$ $\mathrm{sec} /$ point $\left(20 \mathrm{~J} / \mathrm{cm}^{2}\right)$ applied on the bilateral tender points twice/week for 5 weeks. Baseline and final assessment values (after the final treatment session) were compared with the Wilcoxon signed rank test (nonparametric score).

Results: All patients completed the study. Eight of the 9 patients showed significant pain improvement and 6 demonstrated significantly increased trunk mobility ( $\mathrm{P}<0.05$ for both).

Conclusions: LLLT was effective for sacroiliac pain, and this may be due to improvement of the blood circulation of the strong ligaments which support the sacroiliac joint, activation of the descending inhibitory pathway, and the additional removal of irregularities of the sacroiliac joint articular surfaces. Further larger-scale studies are warranted.
\end{abstract}

Key Words: Sacroiliac joint, Low Level Laser Therapy, Sacroiliac joint pain

\section{Introduction}

In the Orthopaedic Department of our university hospital, we often encounter patients with sacroiliac joint pain. In the majority of these cases plane lumbar X-ray films show a clear cause of the lower back pain; however, it is impossible to reach a pathological diagnosis

Addressee for Correspondence:

Takashi Harada

Dept. of Rehabilitation Medicine, Toho University School of Medicine

6-11-1 Omori-nishi, Ota-ku, Tokyo 143-8541 Japan

TEL: +81-3-3762-4151/ FAX: +81-3-3768-6117

E-mail: haradat_3522@yahoo.co.jp of sacroiliac joint pain since the sacroiliac joint is not sufficiently visualized. Traditionally, when patients feel sacroiliac joint pain, it has been considered a secondary partial symptom accompanying lower back degenerative disease.

For patients who complain of chronic sacroiliac joint pain, it is necessary to have a horizontal section CT scan; in reality; however, we are too busy to give routine CT scans. However, some of our outpatients visit us because of sacroiliac joint pain. In the case of chronic sacroiliac joint pain, if pain is the main symp-

Manuscript received: February 15th, 2011 Accepted for publication: May 31st, 2011 
Table 1: Case Outlines

\begin{tabular}{|c|c|c|c|c|c|c|}
\hline \multirow[b]{2}{*}{ Case } & \multirow[b]{2}{*}{ Age } & \multirow[b]{2}{*}{ Sex } & \multicolumn{2}{|c|}{ Pain Score (VAS)* } & \multicolumn{2}{|c|}{ FFD $(\mathrm{cm})^{* *}$} \\
\hline & & & Before treatment & After treatment & Before treatment & After treatment \\
\hline 1 & 56 & $\mathrm{~F}$ & 50 & 50 & 15 & 10 \\
\hline 2 & 54 & $\mathrm{~F}$ & 30 & 20 & 20 & 15 \\
\hline 3 & 59 & $\mathrm{~F}$ & 40 & 40 & 20 & 10 \\
\hline 4 & 39 & M & 70 & 50 & 50 & 40 \\
\hline 5 & 26 & M & 90 & 70 & 50 & 30 \\
\hline 6 & 55 & $\mathrm{~F}$ & 40 & 30 & 10 & 5 \\
\hline 7 & 59 & $\mathrm{~F}$ & 80 & 60 & 25 & 15 \\
\hline 8 & 47 & M & 30 & 20 & 40 & 35 \\
\hline 9 & 59 & $\mathrm{~F}$ & 60 & 30 & 30 & 30 \\
\hline
\end{tabular}

*VAS: Visual Analogue Scale ${ }^{* *}$ FFD: Fingertip-Floor-Distance

tom and there are no other complications, we can watch the clinical course with conservative treatment. We have to think of a differential diagnosis when the patients have sacroiliac joint pain. This requires assessment of the characteristic clinical features of the sacroiliac joint based on case reports in the literature in which a differential diagnosis was required.

Near-infrared low level laser therapy has often been reported as being effective for a variety of chronic musculoskeletal ailments. We therefore designed the present preliminary study to assess the effectiveness of LLLT in improving the pain for patients with chronic sacroiliac joint pain not associated with any of the usual major etiologies.

\section{Subjects and Methods}

\section{Subjects}

The participants in the study were 9 out-patients who visited the Rehabilitation Department at our hospital from 2005 to 2008 (Table 1). There were 4 males and 5 females with an average age of $50.4 \pm 12.6$ years.

\section{Methods}

\section{Etiology}

The patients complained of sacroiliac joint pain, and yet we were able to rule out the usual major disorders, namely lumbar disc hernia, spondylolysis, spinal osteoporosis and metastatic bone tumors of the pelvis. Patients with the symptoms as described below were diagnosed as having sacroiliac joint pain:
- Subjective tenderness was present in the area of their sacroiliac joint (Figure 1).

- Pain developed when the patients' doctors applied pressure to the sacroiliac joint area (Newton Test).

- Pain developed when the patients' doctors distracted or applied pressure to the pelvic girdle.

- Sclerotic change was noted on plain X-ray as a chronic non purulent inflammation around the sacroiliac joint.

- Asymmetry of the sacroiliac joint was seen on a horizontal section CT scan (Figure 2)

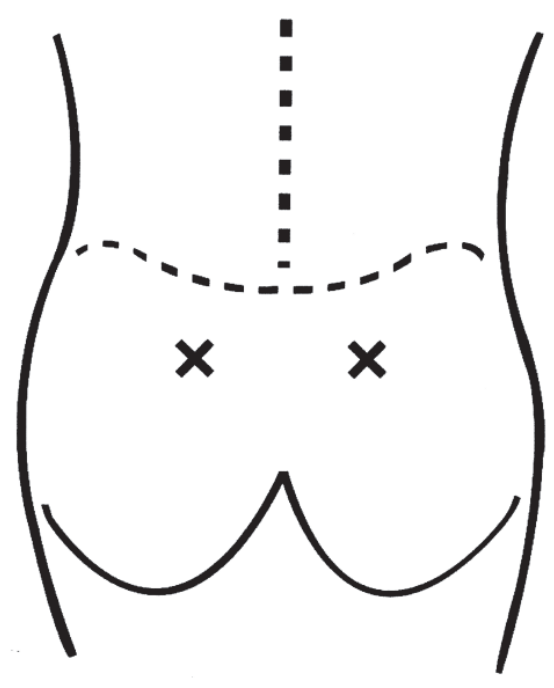

Figure 1: LLLT irradiation part 


\section{available at www.jstage.jst.go.jp/browse/islsm \\ ORIGINAL ARTICLES}

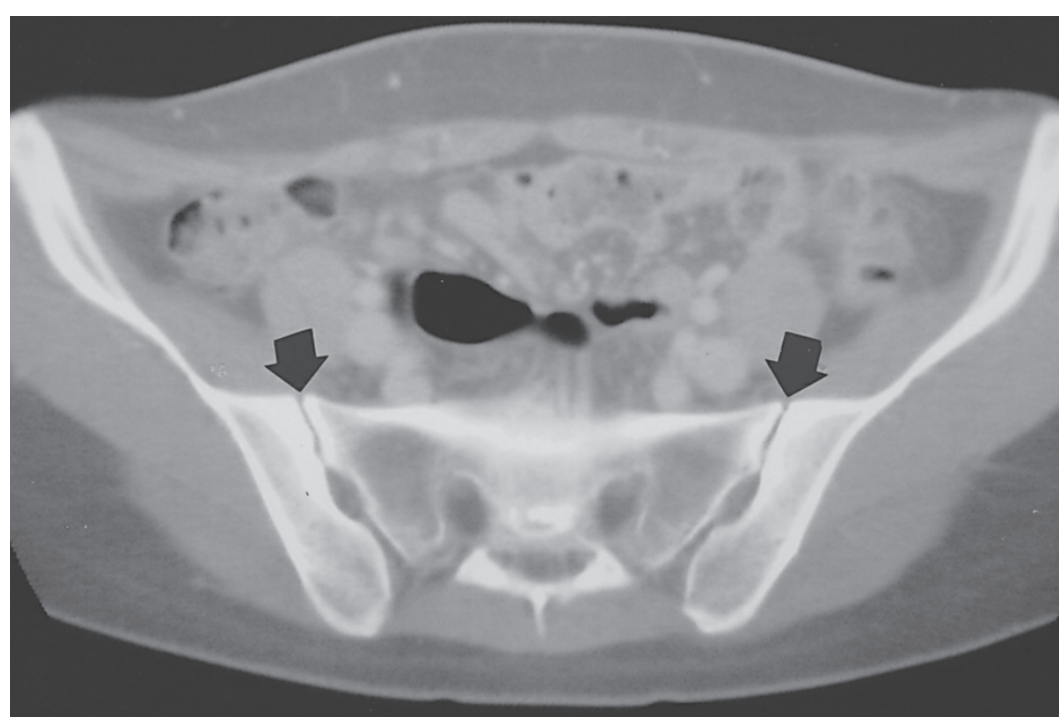

Figure 2: Checking asymmetry of the sacroiliac joint with horizontal section CT imaging

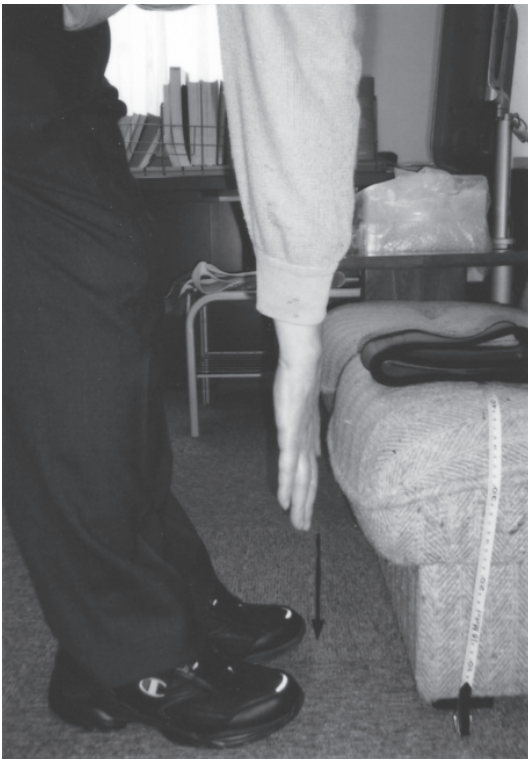

Figure 3: Fingertip Floor Distance : FFD

\section{Evaluation of Pain and Trunk Range of Motion}

Pain was evaluated with a 100-cm long 101-point visual analogue scale (VAS; 100 - maximum pain; 0 - pain free) at baseline and after each laser irradiation session. Trunk range of motion was assessed with the trunk flexion test where we measured the distance in $\mathrm{cm}$ between fingertips and the floor (Fingertip Floor Distance: FFD) (Figure 3) before and after each laser irradiation session.

\section{Laser Therapy Instrument}

We used a gallium aluminum arsenide (GaAlAs) diode low level laser therapy (LLLT) device, the MDL-2001
(Figure $\mathbf{4}$ and Table 2, Matsushita Electric Corporation, Tokyo, Japan). Tender points were irradiated bilaterally with the laser three times for thirty seconds each point (21 $\mathrm{J} / \mathrm{cm}^{2}$ per point), for a total of three minutes for each patient. Each patient underwent this treatment twice a week for 5 weeks, and we performed the final evaluation after the tenth treatment.

\section{Statistical Analysis}

VAS and FFD were measured before treatment started (baseline) and after the final (10 $\left.{ }^{\text {th }}\right)$ LLLT treatment session (final assessment), and those two sets of scores were averaged and compared. We used a $100 \mathrm{~mm}$ VAS and measured the FFD scores in $\mathrm{cm}$. All values were

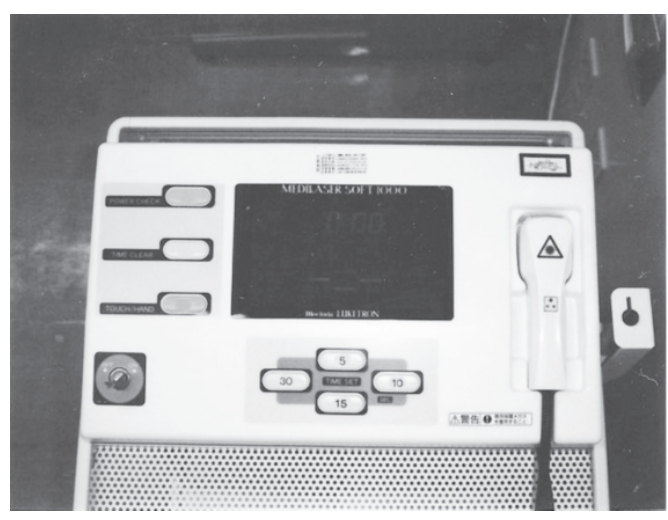

Figure 4: Aspect of Low Level Laser Therapy Device
Table 2: Low Level Laser Therapy Device Specification

\begin{tabular}{|c|c|}
\hline Leaser element & Semiconductor Laser Diode \\
\hline & Ga-Al-As : Gallium-Aluminum-Arsenide \\
\hline Model & MDL-2001 model \\
\hline Manufacturer & Matsushita Electric Corporation,Tokyo, Japan \\
\hline Wavelength & $830 \mathrm{~nm} \pm 15 \mathrm{~nm}$ \\
\hline Output & $1000 \mathrm{~mW} \pm 20 \%$ \\
\hline Mode & Continuous wave mode \\
\hline Irradiation time & $30 \mathrm{sec}$ \\
\hline Energy density & $20.1 \mathrm{~J} / \mathrm{cm}^{2}$ \\
\hline Power supply & $100 \mathrm{VAC}, 50-60 \mathrm{~Hz}$ \\
\hline
\end{tabular}


expressed as the means \pm standard deviation. Comparisons of values at baseline and at the final assessment were performed with the Wilcoxon signed rank test (nonparametric score), with a value of less than $5 \%$ considered significant.

While, with the patients who were under medication, we did not change it pre- and post-irradiation. We kept the same medication using drugs with the similar effect.

The trial was conducted with the approval of the Ethics Committee of the Toho University School of Medicine Institutional Review board (IRB). The purpose and potential outcomes of the trial were explained to all participants, whereafter they gave written informed consent to participate in the study.

\section{Results}

All patients successfully completed the trial. The patients were relatively young, ranging from patients in their twenties to those in their fifties. Changes in VAS and FFD scores before and after treatment are shown in Table 2. We saw a moderate improvement in the baseline and final VAS scores in 8 cases out of the 9 . The FFD scores improved in 8 cases out of 9 , and we could not see any FFD improvement in 1 case.

\section{Complications}

Post-therapy complications, such as lower leg pain, sensory disturbance, bladder or bowel control disturbance were not seen in any patient at any time during the trial.

\section{Results of the Statistical Analysis}

The mean baseline and final VAS scores were $64.4 \pm 21.3$ and $43.3 \pm 16.6$, respectively, and the FFD values were $32.8 \pm 15.4 \mathrm{~cm}$ and $21.7 \pm 13.0 \mathrm{~cm}$, respectively. Both the VAS and FFD baseline and final assessment scores showed significant differences below five percent.

\section{Discussion}

The sacroiliac joints are the "ear" shaped, anatomically unique joints, located between the first and second sacral vertebrae and the iliac bone. The actual movement that occurs within the joint is very small, and this joint does not have a great amount of synovia. ${ }^{1,2}$ Also, from a mechanical standpoint, it is considered to be the skeletal joint taking the greatest load. The weight (all the weight of the head, upper limbs and the trunk) is carried along the spine in a cephalo-caudal direction. At the same time, a ground reaction force is exerted up from the feet to arrive at the sacroiliac joint via the knee and hip joints. At the sacroiliac joint, this weight and the ground reaction force offset each other and become "zero," mechanically speaking. Thus, the sacroiliac joint is considered to play an important part in balancing various bodily movements. When something goes wrong with the sacroiliac joint, abnormalities will develop with the limbs and spinal posture. We consider that this triggers acute and chronic pain in various parts of the body. Hakata, 1,3,4) Murakami, 5) and Kaltenborn ${ }^{2)}$ have pointed out the importance of the role of the sacroiliac joint in causing chronic limb and trunk pain.

In the literature we have additionally encountered some reports on clinical conditions of the sacroiliac joint other than pain, such as injury and deformation, 6, 7) inflammation, ${ }^{8)}$ tumors, 9) and changes caused by aging. ${ }^{10)}$ There are two methods to relieve pain: a conservative approach and surgical intervention. 11,12) As one of the conservative treatments, the ArthroKinetic-Approach (AKA) has been implemented by Hakata, et al., 1,3) with many reports of excellent results. However, we have not tried their AKA treatment method yet because we do not have enough knowledge and experience.

Surgical interventions are indicated in the case of serious injuries and tumor extraction; however, the postoperative results are often poor. Because the sacroiliac joint is located in an anatomically deep position and it is also fixed in place with various strong and powerful ligaments, it cannot be postoperatively precisely replaced to its anatomically correct position. ${ }^{10)}$

In the present preliminary study, laser irradiation of the sacroiliac joint was carried out for the patients whose chronic or acute pain developed due to mechanically-caused bilateral differences of the sacroiliac articular surface. We ascertained that LLLT works effectively for patients without any of the serious diseases as previously mentioned. The LLLT technique is simple and free from harmful side effects; therefore, we can safely administer LLLT treatment in patients of all ages. However, the LLLT treatment effect only lasts for about six hours, so it is important to educate and advise patients about working posture and avoiding unnatural positions for a long time in their everyday activities, in order to prolong the treatment effects.

With the 2 cases with no VAS change and the case with no FFD change, we could not figure out the medical justification. We assume that bracing or AKA treatment can be one of the future treatment policy 
options for these cases.

We assume the mechanism of alleviating sacroiliac joint pain with LLLT irradiation consists of several actions. Irradiation improves blood circulation around the sacroiliac joint, and further blocks the ascending spinothalamic pain transfer mechanism. Algesic neurotransmission is blocked through activation of the descending inhibitory pathway with endorphin, enkephalin and dynorphin synthesis. The tense ligaments which firmly fix the sacroiliac joint in place relax, and the sacroiliac joint adjusts through intracapsular movement, with the result that differences in the joint's bilateral orientation are restored to the correct position.

\section{Conclusions}

In this limited trial population of 9 patients with mechanically-mediated sacroiliac pain treated with 20 $\mathrm{J} / \mathrm{cm}^{2}$ of $830 \mathrm{~nm}$ diode laser LLLT, in 10 sessions over 5 weeks, significant improvements were seen especially in the pain but also in the range of lumbar motion. The results of the trial cannot be accepted as definitive due to the small patient number, but our findings warrant further extended controlled trials with much larger patient populations.

\section{References}

1: Setsuo Hakata: Diagnosis and treatment of pain originating in the sacroiliac joint using arthrokinetic approach. Joint Surgery 22:140-148, 2003.

2: Kaltenborn FM: Manual therapy for the extremity joints, specialized techniques. Olaf Norlis, Bokhandel, Oslo, 1976.

3: Setsuo Hakata, Hiroshi Munesige: Diagnosis and treatment of low back pain using arthrokinematic approach (Hatake!Method). (International Symposium Satellite of $4^{\text {th }}$ Congress of the Pain Clinic:21-25, Tokyo, 2007 (in Japanese)

4: Norisada Sumida: Arthrokinetic approach for the patients with chronic back pain. Orthopaedics 20:46-56, 2007(in Japanese)

5: Eiichi Murakami, Syoichi Kokubun: Sacroiliac pain: its origin and clinical features. Joint Surgery 18:513519, 1999. (in Japanese)

6: Haufe Scott MW, Mork Anthony R: Sacroiliac joint debridement. Photo-medicine and laser surgery 23:596-598, 2007.

7: Chan K, Risnick D: Pelvic instability after bone graft harvesting from posterior iliac crest. Skeletal radiology 30:278-281, 2001.

8: Tsuchiya Kuniyoshi, Bridwell Keith, Kuklo Timothy: Minimum 5-year analysis of L5-S1 fusion using sacropelvic fixation for spinal deformity. Spine 31:303-308, 2007.

9: Whittingham Jones Paul, Hughes Richard: Osteoblastoma crossing the sacro-iliac joint. Skeletal radiology 36:249-252, 2007.

10: Yasumasa Shirai: A study of the sacroiliac joint of sumo wrestlers. Journal Chubu Nippon Seisai :42,6.1999.(in Japanese)

11: Eiichi Murakami, Kyoko Noguchi: Anterior joint fusion for patients with sacroiliac joint pain. Japan Orthopaedic Association 81:104, 2007. (in Japanese)

12: Naonori Asami: Pelvic bone tumor for reconstruction surgery. Neuro surgery, Special Suppl) 14:816819, 2004 (in Japanese). 